\title{
Standardized, Scalable, and Timely Flexible Adeno-Associated Virus Vector Production Using Frozen High-Density HEK-293 Cell Stocks and CELLdiscs
}

\author{
Benjamin Strobel, ${ }^{1}$ Kai Zuckschwerdt, ${ }^{2}$ Gudrun Zimmermann, ${ }^{2}$ Christine Mayer, ${ }^{2}$ \\ Ruth Eytner, ${ }^{2}$ Philipp Rechtsteiner, ${ }^{1}$ Sebastian Kreuz, ${ }^{1}$ and Thorsten Lamla ${ }^{2, *}$ \\ ${ }^{1}$ Research Beyond Borders and ${ }^{2}$ Drug Discovery Sciences, Boehringer Ingelheim Pharma GmbH \& Co. KG, Biberach an der Riss, Germany.
}

Adeno-associated virus (AAV) vectors currently represent the most attractive platform for viral gene therapy and are also valuable research tools to study gene function or establish disease models. Consequently, many academic labs, core facilities, and biotech/pharma companies meanwhile produce AAVs for research and early clinical development. Whereas fast, universal protocols for vector purification (downstream processing) are available, AAV production using adherent HEK-293 cells still requires time-consuming passaging and extensive culture expansion before transfection. Moreover, most scalable culture platforms require special equipment or extensive method development. To tackle these limitations in upstream processing, this study evaluated frozen high-density cell stocks as a ready-to-seed source of producer cells, and further investigated the multilayered CELLdisc culture system for upscaling. The results demonstrate equal AAV productivity using frozen cell stock-derived cultures compared to conventionally cultured cells, as well as scalability using CELLdiscs. Thus, by directly seeding freshly thawed cells into CELLdiscs, AAV production can be easily upscaled and efficiently standardized to low-passage, high-viability cells in a timely flexible manner, potentially dismissing time-consuming routine cell culture work. In conjunction with a further optimized iodixanol protocol, this process enabled supply to a large-animal study with two high-yield AAV2 capsid variant batches $\left(0.6-1.2 \times 10^{15}\right.$ vector genomes) in as little as 4 weeks.

Keywords: cell factories, cell stacks, clinical process development, biotechnology, large-scale AAV production, gene therapy

\section{INTRODUCTION}

DURING THE LAST TWO DECADES, recombinant adeno-associated virus (AAV) vectors have achieved great popularity within biomedical research and remarkable successes in clinical testing, fueling hopes for broad future applications in gene therapy. ${ }^{1}$ In addition to the necessity for easy-to-produce and high-quality vectors during the development of novel gene therapies, AAV vectors are also required for their ever increasing application as a tool in basic and drug discovery research, for instance target validation and disease modeling. ${ }^{2,3}$ Two important aspects that have fostered the broadly applicable production of AAV vectors are the possibility to use conventional, adherently grown HEK-293 cells and inexpensive calcium-phosphate or polyethylenimine (PEI) transfection protocols along with optimized fast purification processes based on iodixanol gradients. ${ }^{4,5}$ Moreover, strategies to suppress transgene expression and related detrimental effects transiently during vector production have been described, thereby facilitating production of difficult-to-produce AAVs. ${ }^{6-8}$

While the steady progress in vector engineering results in a constantly growing list of tailored AAV variants (e.g., for the specific targeting of cell types

${ }^{*}$ Correspondence: Dr. Thorsten Lamla, Drug Discovery Sciences, Boehringer Ingelheim Pharma GmbH \& Co. KG, Birkendorfer Straße 65, Biberach an der Riss, 88397 Germany. E-mail: thorsten.lamla@boehringer-ingelheim.com

(c) Benjamin Strobel, et al., 2019; Published by Mary Ann Liebert, Inc. This Open Access article is distributed under the terms of the Creative Commons Attribution Noncommercial License (http://creativecommons.org/licenses/by-nc/4.0/) which permits any noncommercial use, distribution, and reproduction in any medium, provided the original author(s) and the source are credited. 
or to promote immune evasion), ${ }^{9}$ there is also an increasing challenge for small labs to keep pace in terms of the timely production of various serotypes at high titers, which is required to compare transduction profiles of several AAV variants in vitro or in animal models for instance. Moreover, large amounts of $10^{13}-10^{15}$ vector genomes are usually required to support translational large-animal studies and ultimately clinical trials. One limitation in this regard is the scalability of adherent HEK-293 cell-based AAV production. Several different approaches for upscaling have been evaluated and applied until now, ${ }^{10}$ including roller bottles, ${ }^{11,12}$ multilayered culture systems (e.g., Nunc Cell Factories, Corning CellStacks), ${ }^{13-16}$ and fixed-bed bioreactors. ${ }^{16,17}$ However, these systems either require specialized large-scale equipment (roller bottle incubator, iCellis reactor) and/or extensive method optimization (e.g., to achieve sufficient gas supply in multilayered culture systems), thereby complicating easy method implementation. The recently developed round-shaped CELLdiscs (Greiner Bio-One) represent an alternative multilayered cell culture system whose central gas supply channel and liquid port improve both handling (e.g., less foaming) and (at least in the authors' hands) cell viability compared to alternative multilayer culture systems. One aspect of this study therefore was to evaluate CELLdiscs for the production of recombinant $\mathrm{AAV}$ vectors.

A second factor contributing to longer time lines, little flexibility, and high workload during vector production is the necessity for extensive HEK-293 cell culture expansion prior to seeding cells for production (Fig. 1a). More specifically, for a standard small-scale laboratory AAV batch produced by transfection of (in the authors' lab) forty $15 \mathrm{~cm}$ cell culture dishes, approximately 100 million cells are seeded 3 days before transfection, requiring five to eight T175 flasks grown to $70-80 \%$ confluency prior to seeding. Likewise, Lock et al. described their expansion strategy, starting from sixteen $15 \mathrm{~cm}$ dishes over two 10-layer culture systems to finally seed six 10-layer cell stacks for large-scale production. ${ }^{18}$ Besides the need for media, culture flasks, and time, a side effect of routine cell culture passaging and expansion is the variability with regard to culture passage and associated decrease in AAV productivity in higherpassage cells-an often-heard warning in the field that was lately also proven experimentally. ${ }^{19}$

To enable standardization, professional services offering large-scale cell expansion, automated aliquot dispensing, and controlled cryopreservation are available and well established in biopharma- ceutical production (e.g., to generate master and working cell banks). Moreover, to enable quick performance and to standardize conditions, assay-ready frozen cells are often used as a source for large cellular screening campaigns. Rethinking these concepts, it was speculated that frozen cell stocks might also simplify AAV production. Therefore, the second aspect of this study was to evaluate commercially available, high-density (e.g., 60 million cells per vial), frozen cell stocks as a ready-to-seed source of high-viability, low-passage HEK-293 producer cells.

Following a side-by-side comparison of AAV vector production efficiency using normally cultured/ passaged versus frozen cell stock-derived cultures, the study evaluated the scalability of the culture dish-based protocol to multilayered CELLdiscs. It was demonstrated that AAV production based on the direct seeding of a single vial of frozen cells into a 16-layer, 4,000 $\mathrm{cm}^{2}$ CELLdisc (Fig. 1b) is similarly efficient as an equally sized AAV production run conducted using conventionally cultured cells and culture dishes. Finally, this approach was used to supply a nonhuman primate (NHP) study with two AAV vector batches of $6 \times 10^{14}$ and $1 \times 10^{15}$ vector genomes (VG), respectively, whereby production, purification, and quality control (QC) was carried out in a standard lab within a time frame of 4 weeks. Thus, this method, together with a further optimized variant of a previously reported iodixanol protocol, ${ }^{4}$ allows for the rapid, scalable, timely, flexible, and standardized production of AAV vectors in adherent HEK-293 cells.

\section{METHODS}

\section{Cells and culture conditions}

HEK-293H cells were purchased from Thermo Fisher Scientific (cat. no. 11631017) and cultured in Dulbecco's modified Eagle's medium (DMEM) + GlutaMAX-I + 10\% fetal calf serum (FCS; Gibco/ Thermo Fisher Scientific). Multi-well plates were purchased from Thermo Fisher Scientific/Nunc, and $15 \mathrm{~cm}$ culture dishes were obtained from Sarstedt. CELLdiscs were purchased from Greiner Bio-One.

\section{Preparation of frozen high-density HEK-293 cell stocks}

Frozen high-density HEK-293H cell stocks ("assay ready frozen instant cells”) were produced by acCELLerate $\mathrm{GmbH}$ at requested cell densities of either 50 (standard) or 60 (adapted for CELLdisc format) million cells per $2 \mathrm{~mL}$ cryovial using the following protocol. Cells were thawed and cultivated in DMEM (Sigma-Aldrich) supplemented with $10 \%$ FBS (Corning) and $2 \mathrm{mM}$ L-Glutamine (PAN Biotech) 


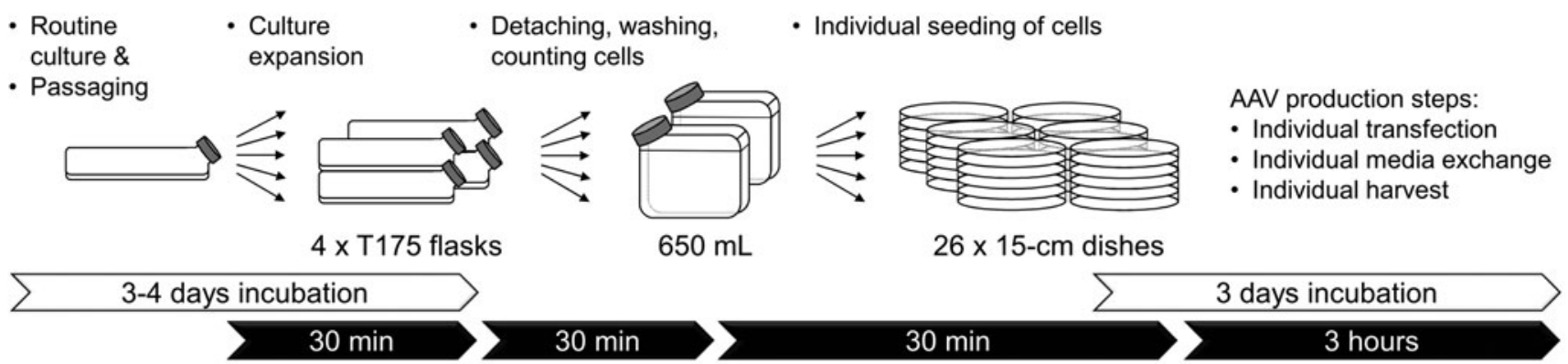

b Frozen high-density cell stocks

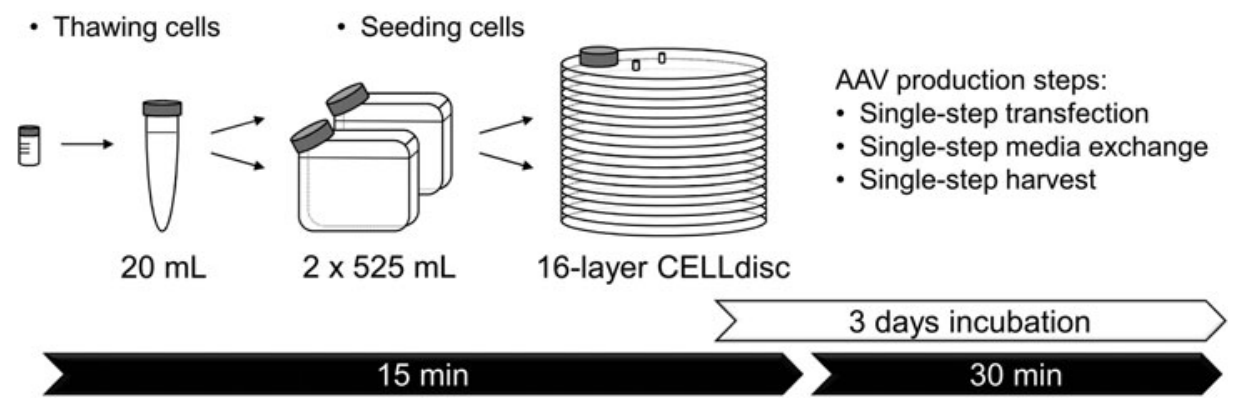

Figure 1. Process steps and time requirement for adeno-associated virus (AAV) "upstream" processing at $4,000 \mathrm{~cm}^{2}$ scale. (a) For lab-scale AAV manufacturing, HEK-293 cells are regularly passaged and expanded 3-4 days prior to seeding for AAV production, requiring approximately $1.5 \mathrm{~h}$ of hands-on work in total (black arrows). AAV production (i.e., transfection, media change, and cell harvest) takes approximately $3 \mathrm{~h}$ of hands-on-work when using regular cell culture systems such as culture dishes or flasks. (b) The frozen high-density cell-based protocol relies on thawing and resuspension of high-density frozen cells, followed by direct seeding in either culture dishes or CELLdiscs (approximately $15 \mathrm{~min}$ of hands-on work), thereby eliminating the need for culture expansion and associated steps. The possibility of conducting transfection, media change, and harvest in a single step drastically reduces the hands-on work to approximately 30 min in total.

at $37^{\circ} \mathrm{C}$ in a humidified atmosphere with $5 \% \mathrm{CO}_{2}$. To maintain the cells in a logarithmic growth phase, they were kept at a density of $<2.5$ cells $/ \mathrm{cm}^{2}$. For passaging, cells were gently detached using Accutase (Sigma-Aldrich) and reseeded at a density of 20,000 cells $/ \mathrm{cm}^{2}$. For the preparation of 200 vials, cells were expanded in T-flasks and CellSTACKs (Corning) to an estimated minimum yield of $1.2 \times 10^{10}$ cells. The cells were detached with Accutase, washed once in culture medium, and re-suspended in culture medium containing 5\% dimethyl sulfoxide for cryoprotection at a density of $3.4 \times 10^{7}$ cells $/ \mathrm{mL}$. Next, $1.8 \mathrm{~mL}$ of the cell suspension (i.e., 60 million cells) was automatically dispensed into 200 cryovials (Nunc) using an XSD-Biofill decapping and filling device (FluidX). Finally, the cells were frozen in a Cryomed 7452 controlled rate freezer (Thermo Fisher Scientific) at a cooling rate of $1^{\circ} \mathrm{C} / \mathrm{min}$ and transferred to storage in the vapor phase of liquid nitrogen.

\section{AAV production at 24-well scale, titration in crude lysate, and transduction assay}

HEK-293 cells $(n=30,000)$ were seeded in DMEM $+10 \%$ FCS per well of a 24 -well plate 3 days prior to transfection. The cells were transfected using the calcium phosphate method, as previously described, ${ }^{4}$ with a total of $1.0 \mu \mathrm{g}$ DNA, comprising equimolar amounts of rep/cap, helper, and transgene plasmids. Specifically, cells were transfected with a pAAV-CMV-eGFP-SV40pA transgene plasmid and either pDP1, pDG, pDP5, pDP6, or pDP8 (Plasmid Factory) or an AAV9-rep/cap plasmid along with the pHelper plasmid (Applied Biosystems). For benzonase control samples, cells were transfected with the enhanced green fluorescent protein (eGFP) transgene plasmid and pHelper only. Six hours after transfection, the media was replaced, and cells were incubated for $72 \mathrm{~h}$ at $37^{\circ} \mathrm{C}$. Cells were then detached by pipetting and pelleted by centrifugation at $1,000 \mathrm{~g}$ for $3 \mathrm{~min}$. After carefully removing the supernatant, $500 \mu \mathrm{L}$ phosphatebuffered saline (PBS)-MK $(1 \times \mathrm{PBS}, 1 \mathrm{mM} \mathrm{MgCl}$, and $2.5 \mathrm{mM} \mathrm{KCl}$ ) were added to the cells, which were then lysed by three freeze/thaw cycles using liquid nitrogen and a $37^{\circ} \mathrm{C}$ water bath. Cell debris was pelleted by centrifugation at $10,000 \mathrm{~g}$ for $3 \mathrm{~min}$. AAV titer determination in cell lysate was conducted largely as described recently. ${ }^{20}$ Briefly, $10 \mu \mathrm{L}$ of lysate were pipetted onto a 96 -well plate, and $2 \mu \mathrm{L}$ (50 IU) benzonase was added, mixed, and 
incubated at $37^{\circ} \mathrm{C}$ overnight (for at least $15 \mathrm{~h}$ ) in a polymerase chain reaction (PCR) cycler, followed by benzonase inactivation at $75^{\circ} \mathrm{C}$ for $30 \mathrm{~min}$. Following the addition of $7.5 \mu \mathrm{L}$ (6 IU) Proteinase $\mathrm{K}$ (Thermo Fisher Scientific) and incubation for $2 \mathrm{~h}$ at $56^{\circ} \mathrm{C}$, Proteinase was inactivated at $95^{\circ} \mathrm{C}$ for 30 min. Finally, samples were diluted 1:20 in water and used for quantitative PCR-based detection of AAV vector genomes using a cytomegalovirus (CMV) promoter-specific primer/probe set.

For the assessment of AAV bioactivity, 10, 25, or $50 \mu \mathrm{L}$ of centrifuged lysate (before benzonase addition) was added to HEK-293 cells on 96-well plates, followed by incubation at $37^{\circ} \mathrm{C}$ for $72 \mathrm{~h}$. Cells were then detached, re-suspended in PBS $+10 \%$ FCS, and analyzed for GFP expression by flow cytometry (10,000 cells per condition).

\section{AAV production in culture dishes and CELLdiscs}

Cells $\left(2.4 \times 10^{6}\right.$ and $\left.6 \times 10^{7}\right)$ were seeded per $15 \mathrm{~cm}$ dish and 16-layer CELLdisc $\left(4,000 \mathrm{~cm}^{2}\right)$ in 25 and 1,050 mL DMEM + GlutaMAX-I + 10\% FCS 3 days prior to transfection, respectively. In the case of frozen cell stocks, a vial with $6 \times 10^{7}$ cells was rapidly thawed at $37^{\circ} \mathrm{C}$, wiped with an ethanol-soaked cloth, and added to $20 \mathrm{~mL}$ prewarmed culture medium. Next, $10 \mathrm{~mL}$ of this cell suspension was added to each of two bottles of prewarmed $525 \mathrm{~mL}$ culture medium and gently mixed by rotating. Two bottles of cell suspension were then poured into one CELLdisc and equally spread on all layers, following the handling instructions provided with the CELLdiscs, and incubated for $72 \mathrm{~h}$ at $37^{\circ} \mathrm{C}$. For transfection, $0.5 \mu \mathrm{g}$ of total plasmid DNA were used per square centimeter of growth area in an equimolar ratio (i.e., under consideration of plasmid size). $\mathrm{pDG}$, $\mathrm{pDP} 8$, or AAV9, AAV2-BR1, or AAV2-L1 (termed AAV2-ESGHGYF in the original publication $)^{21,22}$ rep/cap plasmids were mixed with pHelper (Applied Biosystems) and an AAV2 ITR-containing CMV-eGFP or CAG-eGFP plasmid. For one 16 -layer $/ 4,000 \mathrm{~cm}^{2}$ CELLdisc, the DNA was then mixed with $69 \mathrm{~mL} 300 \mathrm{mM} \mathrm{CaCl}_{2}$ and mixed by rotation. This mix was added dropwise to $69 \mathrm{~mL} 2 \times$ HBS buffer, $\mathrm{pH} 7.0$ (Alfa Aesar/ Thermo Fisher Scientific). After incubation for approximately $2 \mathrm{~min}$ and visual confirmation of slight turbidity, the solution was added to a medium bottle with $5 \%$ FCS $(525 \mathrm{~mL})$. If multiple CELLdiscs were to be transfected, each transfection mix was prepared separately immediately before addition. The CELLdisc medium was then replaced with the $663 \mathrm{~mL}$ transfection medium and incubated for 4-6h. The transfection medium was then replaced again with $1,050 \mathrm{~mL}$ fresh culture medium (5\% FCS; optionally supplemented with pen/strep) and incubated for $72 \mathrm{~h}$. Transfection of cell dishes overall followed the same approach, but with direct addition of transfection mix to the dishes, as described in detail before. ${ }^{4}$ Four to six hours after transfection, the transfection medium was replaced with fresh medium (5\% FCS), and cells were incubated for $72 \mathrm{~h}$ until harvest.

\section{AAV harvest and cell lysis}

For harvest of CELLdiscs, one $500 \mathrm{~mL}$ centrifugation tube was prepared with $7 \mathrm{~mL} 0.5 \mathrm{M}$ EDTA and then filled with the cell supernatant from the CELLdisc. The residual supernatant was poured into a second tube. The $7 \mathrm{mM}$ EDTA-containing supernatant was then poured back into the CELLdisc and incubated for $5 \mathrm{~min}$ at room temperature (RT) to detach the cells, which was supported by tapping against and tumbling the CELLdisc. After harvesting the cells, the second tube with supernatant was used to flush the CELLdisc. Cells from both tubes were pelleted at $800 \mathrm{~g}$ and $4^{\circ} \mathrm{C}$ for $15 \mathrm{~min}$. Cell pellets of one CELLdisc were re-suspended in $26 \mathrm{~mL}$ lysis buffer (1 M NaCl, $50 \mathrm{mM}$ Tris, $10 \mathrm{mM}$ $\mathrm{MgCl}_{2}, 0.001 \%$ Pluronic F-68; Thermo Fisher Scientific), $\mathrm{pH} 8.5,4^{\circ} \mathrm{C}+$ freshly added $1,300 \mathrm{IU}$ (i.e., $0.325 \mathrm{IU} / \mathrm{cm}^{2}$ ) salt active nuclease (Serva), and $1 \times$ HALT protease inhibitor (Thermo Fisher Scientific) and applied to three freeze/thaw cycles using liquid nitrogen and a $37^{\circ} \mathrm{C}$ water bath for approximately 10 min each. Following the last thaw cycle, another 1,300 IU salt active nuclease was added, and the lysate was incubated in a $37^{\circ} \mathrm{C}$ shaking incubator for $60 \mathrm{~min}$. Cell debris was then pelleted at 2,500 $\mathrm{g}$ at RT for $15 \mathrm{~min}$, and the AAV-containing supernatant was used for further purification. In essence, the same protocol was used for cell dishes. However, for detachment, $350 \mu \mathrm{L} 0.5 \mathrm{M}$ EDTA was directly added to the culture dish. If fewer than 26 dishes (equaling one 16-layer CELLdisc) were used, reagents and volumes were reduced accordingly.

\section{AAV purification (polyethylenglycol precipitation, iodixanol gradient, ultrafiltration)}

One quarter volume of a $40 \%$ polyethylenglycol (PEG)-8000 solution (i.e., 8\% final concentration) was added to the AAV-containing supernatant obtained after cell lysis and centrifugation (described above), mixed well, and incubated on ice for at least $3 \mathrm{~h}$. After centrifugation at $2,500 \mathrm{~g}$ and $4^{\circ} \mathrm{C}$ for $30 \mathrm{~min}$, the supernatant was discarded, and the pellet was quickly centrifuged again to remove residual supernatant fully. The pellet was then taken up in $14 \mathrm{~mL}$ resuspension buffer ( $1 \mathrm{M} \mathrm{NaCl}, 50 \mathrm{mM}$ 
Tris, $0.001 \%$ Pluronic F-68, $\mathrm{pH}$ 8.0) and resuspended thoroughly using a $10 \mathrm{~mL}$ serological pipette. To dissolve pellet fragments fully, the mixture was then incubated overnight (16-24h) at $4^{\circ} \mathrm{C}$ on an overhead rotator $(20-25 \mathrm{rpm})$. The solution was then vortexed and centrifuged at 2,500 $\mathrm{g}$ and $4^{\circ} \mathrm{C}$ for 30 min before the AAV-containing supernatant was filled into a $39 \mathrm{~mL}$ Beckman Coulter QuickSeal tube using a syringe.

An iodixanol step gradient was then prepared by successively underlaying $9 \mathrm{~mL} 15 \%, 6 \mathrm{~mL} 25 \%$,

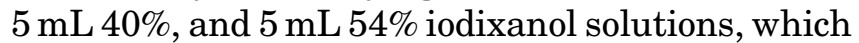
were prepared as described before ${ }^{4}$ but with $0.001 \%$ Pluronic F-68 added to all solutions. If necessary, the tube was filled up with re-suspension buffer and sealed. The gradient was centrifuged for $1 \mathrm{~h} 15 \mathrm{~min}$ at $350,000 \mathrm{~g}$ and $18^{\circ} \mathrm{C}$ in a $70 \mathrm{Ti}$ fixed-angle rotor and Optima XPN-80 ultracentrifuge (Beckman Coulter). The tube was then punctured at the top (to allow for air inflow) and at the bottom using a $20 \mathrm{G}$ needle. The tube was then placed on a $15 \mathrm{~mL}$ tube (while closing the ventilation hole with a finger), and $4 \mathrm{~mL}$ of the lowest iodixanol phase (54\%) was collected for disposal by opening the ventilation hole. Using an additional $15 \mathrm{~mL}$ tube, the following $3.5 \mathrm{~mL}$ was collected as the AAV target fraction, whereas the residual fluid was discarded.

An Amicon Ultra-15 MWCO 100,000 ultrafiltration tube (Merck Millipore) was equilibrated twice with $1 \times \mathrm{PBS}$ and once with AAV formulation buffer $(1 \times \mathrm{PBS}, 1 \mathrm{mM} \mathrm{MgCl} 2,2.5 \mathrm{mM} \mathrm{KCl}, 10 \%$ glycerol, $0.001 \%$ Pluronic F-68, $\mathrm{pH} 7.4$, sterile-filtered). The $3.5 \mathrm{~mL}$ AAV target fraction was then added to the tube and filled up to $15 \mathrm{~mL}$ using formulation buffer. It was then centrifuged at $1,000 \mathrm{~g}$ and $20^{\circ} \mathrm{C}$ until a retentate volume of about $1.5 \mathrm{~mL}$ was achieved. The tube was filled up to $15 \mathrm{~mL}$ again and centrifuged until the desired final product volume (usually around $1 \mathrm{~mL}$ ) was achieved. Finally, the solution was sterile filtered using Ultrafree-CL filter tubes (Merck Millipore), aliquoted, snap frozen in liquid nitrogen, and stored at $-80^{\circ} \mathrm{C}$.

\section{Titration and quality control}

For the detection of AAV vector genomes, viral DNA was isolated using the ViralXpress nucleic acid extraction kit (Merck Millipore) and diluted 1:100 in water. Quantitative PCR was conducted using the $2 \times$ QuantiFast probe PCR master mix (Qiagen) and a primer/probe set specifically binding a sequence segment of the CMV promoter (AAV2-L1-CMV-eGFP) that is also contained in the CAG promoter (AAV2-BR1-CAG-eGFP). The respective plasmids were used to prepare a standard curve for quantification by serial 1:5 dilutions. AAV productivity (VG/cell) was calculated based on the number of cells present at the time of transfection, determined to be $182,000( \pm 24,000)$ cells $/ \mathrm{cm}^{2}$ in a $4,000 \mathrm{~cm}^{2}$ CELLdisc, in a one-time experiment based on five measurements, which was then extrapolated and used as reference for follow-up experiments at different scales. Cryo electron micrographs were prepared at Vironova and inspected visually to determine empty-to-full particle ratios by manual counting. For sodium dodecyl sulfate polyacrylamide gel electrophoresis (SDSPAGE), samples were denatured in lane marker reducing sample buffer (Thermo Fisher Scientific) at $95^{\circ} \mathrm{C}$ and run on a NuPAGE $4-12 \%$ Bis-Tris mini gel (Thermo Fisher Scientific). Proteins were then stained, using the SYPRO Ruby Protein Gel Stain (Thermo Fisher Scientific) as per protocol and visualized under UV light.

\section{RESULTS AND DISCUSSION \\ Evaluation of frozen cell stocks \\ as a ready-to-seed producer cell source}

HEK-293 cell-based protocols used to date usually rely on the expansion of regularly cultured cells prior to seeding of the cells for AAV production, typically 3 days prior to transfection (Fig. 1a). It was speculated that 3 days would be sufficient to allow frozen cell stock-derived, high-density producer cells to recover, adapt to the culture conditions, and grow to the desired confluency of approximately 70-80\%. To evaluate this approach, a conventional HEK-293H cell stock was obtained, and the cells were brought to culture. Part of the cells were then continued in routine culture and passaging, whereas parts were sent to acCELLerate $\mathrm{GmbH}$ for large-scale expansion and generation of high-density (i.e., 50 million cells per vial) "assay ready frozen instant cell" stocks. Either regularly cultured HEK-293 cells or freshly thawed cell stock-derived cells were then seeded at equal cell counts into 24-well plates and, 3 days after, transfected for AAV-eGFP production.

Examination of cells prior to transfection did not show any morphological differences between frozen cell stock- and culture-derived cells, as well as similar confluency (Fig. 2a). Also, transfection efficiency, assayed by measuring GFP fluorescence signal, showed no significant differences between cultured and cell stock-derived cells (Fig. 2b). Importantly, the AAV titers measured in crude lysate clearly showed that AAV-eGFP vectors of all six tested serotypes $1,2,5,6,8$, and 9 were produced at similar efficiency in both culture- and frozen stockderived producer cells (Fig. 2c). More specifically, 
a

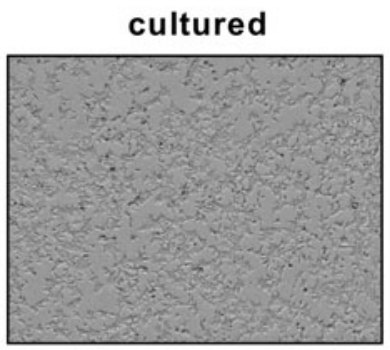

frozen

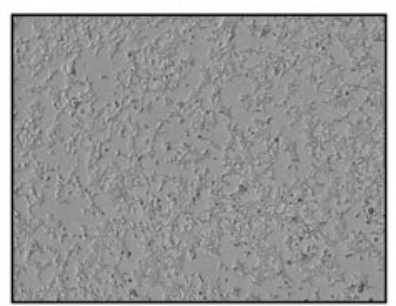

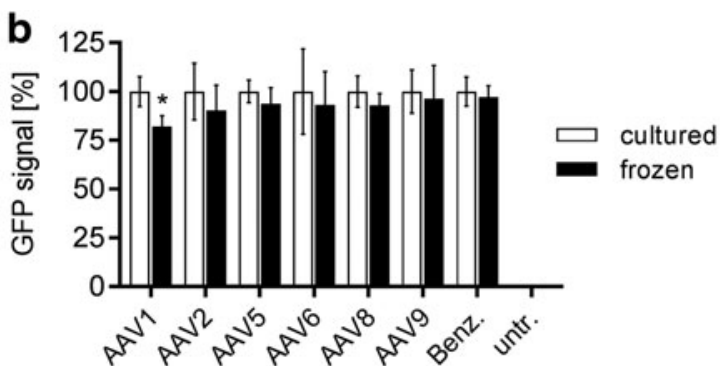

C

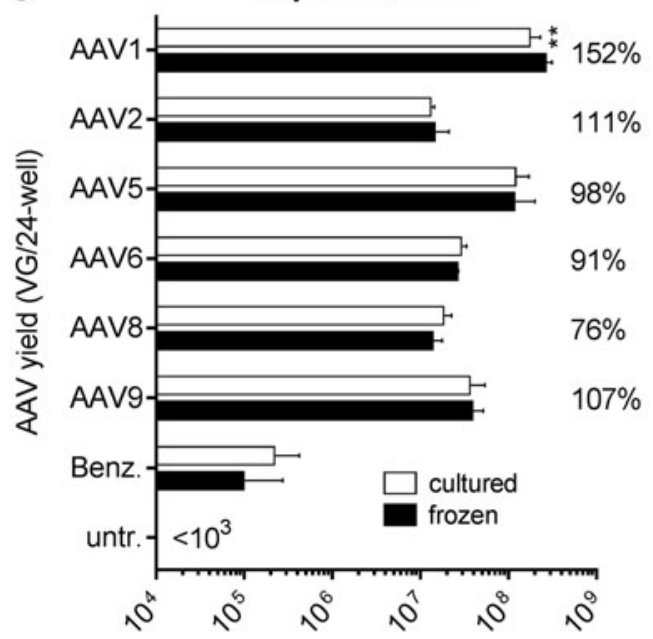

experiment 2

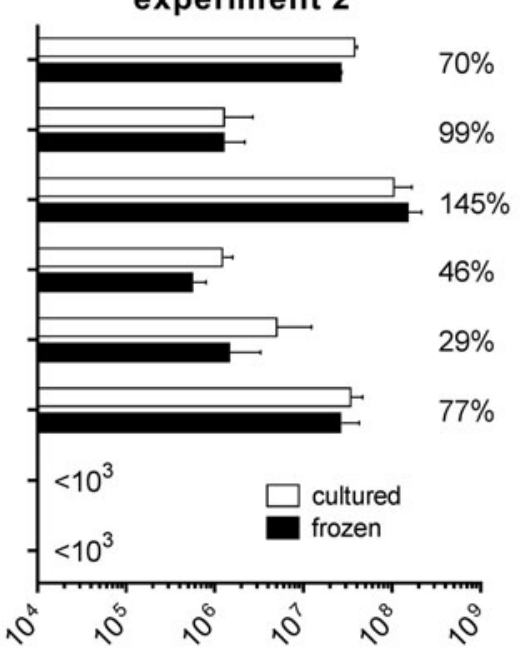

experiment 3

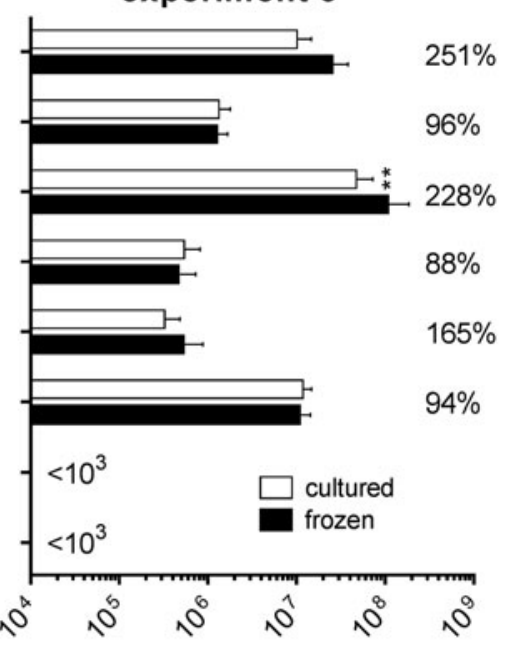

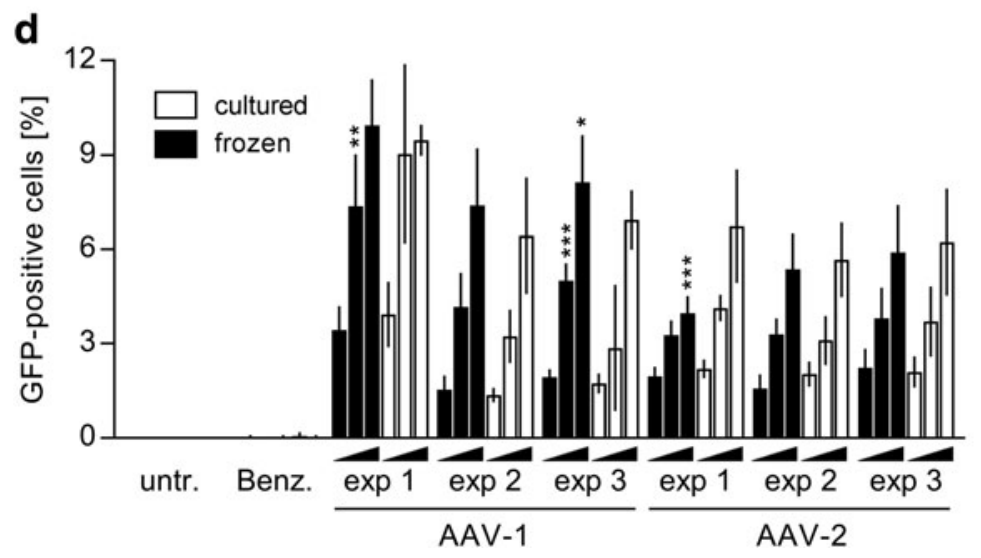

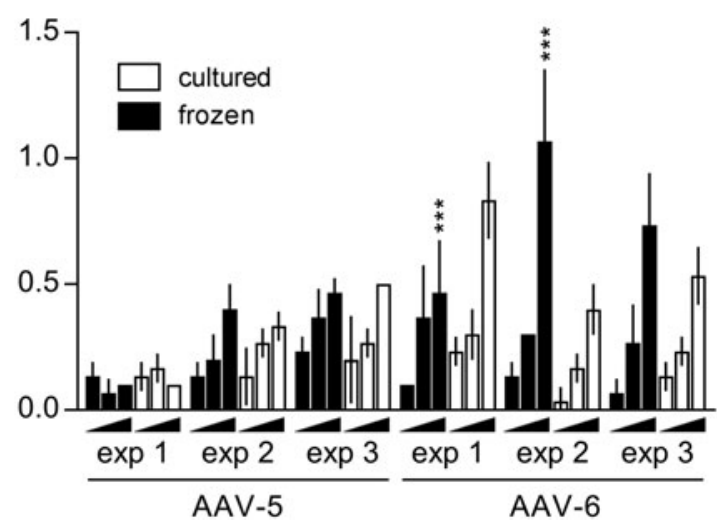

Figure 2. AAV production using frozen cell stock-derived cultures is similarly efficient as in conventionally cultured cells. (a) Bright-field micrographs (10 $\times$ ) of HEK-293 cells $72 \mathrm{~h}$ after seeding of conventionally passaged cells ("cultured") or cells derived from frozen cell stocks. (b) Direct green fluorescent protein (GFP) fluorescence of cells $72 \mathrm{~h}$ after transfection for AAV-GFP production $\left(n=3, M \pm S D\right.$, $\left.{ }^{*} p<0.05\right)$. (c) AAV yield (vector genomes [VG]) per well of a 24-well plate. The percentage indicates the AAV yield obtained from cultures based on frozen cell stocks relative to conventional cultures. Three independent experiments with three biological replicates each are shown. $M \pm S D ;{ }^{* *} p<0.01$. (d) Transduction efficiency of AAVs obtained from the experiments shown in (c). HEK-293 cells were seeded on 96-well plates and transduced using 10, 25, or $50 \mu \mathrm{L}$ of crude lysate. GFP-positive cells were quantified by flow cytometry $(10,000$ cells $) 72 \mathrm{~h}$ after addition of crude lysate. $n=3$ biological replicates. $M \pm S D_{;}^{*} p<0.05,{ }^{* *} p<0.01,{ }^{* * *} p<0.001$. Benz., benzonase control; untr., untreated.

three independent rounds of AAV production revealed following yields obtained from frozen cellderived cultures in comparison to conventionally cultured cells: AAV1: $158 \pm 87 \%$; AAV2: $102 \pm 6 \%$; AAV5: $157 \pm 71 \%$; AAV6: $75 \pm 29 \%$; AAV8: $90 \pm 60 \%$; AAV9: $93 \pm 16 \%$. On average, production using frozen cell-derived cultures yielded $112 \%$ of the titers obtained from conventional cultures (differ- ence not statistically significant), which allows for the conclusion that AAV production efficiency was similar in both culture- and frozen cell stockderived cells.

To prove that the produced AAVs were similarly bioactive, HEK-293 cells were transduced with increasing volumes of crude lysate-derived AAVs from the three different batches, and GFP-positive 
cells were quantified by flow cytometry 3 days after. Whereas at a few doses statistically significant but non-systematic differences were observed, most wells showed equal transduction efficiency for AAVs $1,2,5$, and 6 , independent of the culture type from which they were derived (Fig. 2d). AAV8 and 9 vectors did not lead to sufficient transduction in this crude lysate-based experiment due to their very limited HEK-293 transduction efficiency at low multiplicity of infection and are therefore not shown. In summary, the results demonstrate that vectors produced in frozen cell-derived cultures behaved indistinguishably from vectors produced conventionally.

\section{Upscaling and frozen cell stock-based production in CELLdiscs}

To address the issue of cumbersome culture dish handling and scalability, next the possibility of producing AAVs in the newly available CELLdisc culture system was assessed. Following linear upscaling calculations according to growth area and some minor optimization of cell counts, 60 million cells was established as a suitable cell number for seeding of HEK-293 cells into a $4,000 \mathrm{~cm}^{2}$ CELLdisc 3 days prior to transfection. While cells were seeded in medium containing $10 \%$ serum, the serum concentration was reduced to $5 \%$ in all following steps (i.e., transfection, production) to avoid excessive foam formation when pouring the media into or out of the CELLdiscs. Moreover, because the combined use of frozen cell stocks and CELLdiscs would offer dramatic improvement in terms of handling time, standardization, and scalability, frozen cell stock-derived cells were also incorpo- rated in this side-by-side set of experiments. Notably, whereas for 26 cell dishes $\left(3,952 \mathrm{~cm}^{2}-\right.$ approximately equaling one 16 -layer $/ 4,000 \mathrm{~cm}^{2}$ CELLdisc), each of the four main process steps (cell seeding, transfection, media exchange, and harvest) has to be carried out individually for each dish by pipetting (i.e., $4 \times 26=104$ pipetting steps), CELLdiscs allow for much easier and faster handling by simply pouring the whole volume of cells, culture medium, transfection mix, EDTA solution, and detached cells, respectively (Fig. 1).

Following side-by-side production of AAV2eGFP vectors and quantification of the final iodixanol-purified product, similar production efficiencies were observed with all three approaches, that is, conventionally cultured cells grown in twenty-six $15 \mathrm{~cm}$ cell dishes, cultured cells grown in a $4,000 \mathrm{~cm}^{2}$ CELLdisc, and, most importantly, frozen cell stock-derived cells directly seeded and grown in a CELLdisc (Fig. 3). While some differences in absolute AAV yields were observed between the four sets of side-by-side experiments (see \#1-\#4 in Fig. 3)-which were produced independently, partly by different operators and on different days-the overall yields between the three production methods were similar across all batches. Moreover, cell viability measurements in surrogate plates revealed equal viability on the day of transfection, that is, $72 \mathrm{~h}$ after seeding of cultured cells in dishes $(97.33 \%)$ and frozen stock-derived cells in CELLdiscs (97.28\%). To complement these results further, additionally four independent batches of both AAV8 and AAV9 were produced, which confirmed the finding that AAV yields obtained from frozen cell stock-derived
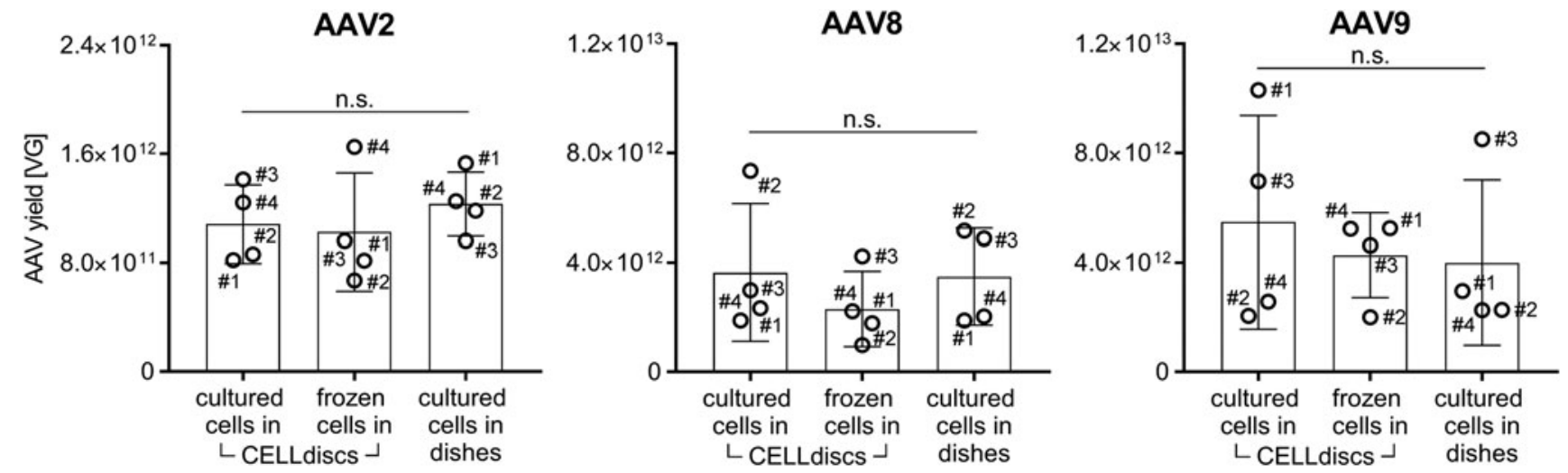

Figure 3. AAV production based on frozen cell stock-derived cells can be upscaled to CELLdiscs. For each batch, HEK-293 cells were seeded from either conventionally cultured cells or from a vial of high-density frozen cells $\left(60 \times 10^{6}\right)$ into a 16 -layer $4,000 \mathrm{~cm}^{2}$ CELLdisc or twenty-six $15 \mathrm{~cm}$ culture dishes $\left(\sim 3,952 \mathrm{~cm}^{2}\right)$ and transfected for AAV-GFP production. Seventy-two hours after transfection, cells were harvested, and AAVs were purified by a combined polyethylenglycol (PEG) precipitation and iodixanol ultracentrifugation protocol (see text and Fig. 4). Vectors were quantified by quantitative polymerase chain reaction (qPCR), and AAV yields are presented as VG for four independently produced AAV batches (numbered \#1-\#4) per serotype. $M \pm S D$. n.s., not significant. 
CELLdisc cultures equaled those from normally cultured cells grown in individual cell dishes (Fig. 3).

\section{AAV large-scale production using the "one vial per CELLdisc" approach}

Having successfully set up this new production protocol, finally it was applied in the manufacturing of two large-scale AAV batches for a NHP biodistribution study of AAV2-BR1 and AAV2-L1 (termed AAV2-ESGHGYF in the original publication $^{22}$ )—two peptide insertion variants of AAV2 that were shown to specifically target the brain (BR1) and lung (L1) after systemic administration in mice. ${ }^{21,22}$ Of note, AAV2 vectors usually yield lower titers than other serotypes (e.g., AAV8 and AAV9; Fig. 3 and Vandenberghe et $a l .{ }^{23}$ ), and engineered capsids often also tend to be produced less efficiently than non-engineered capsids, possibly due to the impact of mutations/insertions on capsid stability and packaging, ${ }^{24}$ therefore posing additional challenges for high-titer manufacturing.

For AAV2-BR1 and -L1 manufacturing, over a time frame of 4 weeks, a total of 28 CELLdiscs were transfected per AAV2 variant. Cells were detached using EDTA, pelleted by centrifugation, and lysed by a high salt osmotic shock and three freeze/thaw cycles. DNA was simultaneously digested using a salt-active nuclease, and cell debris was removed by centrifugation. An optimized version of the previously reported iodixanol protocol, ${ }^{4}$ further improved for throughput/loading capacity, purity, and ease of handling, was then used for AAV purification (see Fig. 4 and Methods section for details). Briefly, as a pre-purification step, AAV particles were pelleted by PEG-8000 precipitation, thereby removing a significant portion of cellular protein, which further facilitates purification efficiency by the subsequent $1 \mathrm{~h}$ iodixanol gradient ultracentrifugation step. Contrary to published protocols, which incomprehensibly recommend isolating AAVs by puncturing the side of the centrifugation tube, ${ }^{25}$ thereby risking contamination with empty particles and host-cell protein from the upper $40 \%$ and $25 \%$ iodixanol phases, AAVs were isolated from the bottom of the tube by simply discarding the first $4 \mathrm{~mL}$ outflow and collecting the following $3.5 \mathrm{~mL}$ as a target fraction. AAVs were then concentrated and formulated in storage buffer by ultrafiltration, sterile-filtered, aliquoted, and snap-frozen. Because isolated AAVs from two CELLdiscs were finally concentrated using one common ultrafiltration tube, 14 batches were produced, quantitated, and quality controlled for each
- EDTA-mediated harvest

- Pelleting of cells $(800 \mathrm{xg}, 15 \mathrm{~min})$

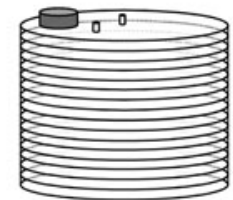

$$
\begin{aligned}
& \text { - Lysis by high-salt and } \\
& 3 \times \text { freeze/thaw } \\
& \text { - Nuclease DNA digestion } \\
& \left(1 \mathrm{~h}, 37^{\circ} \mathrm{C}\right) \\
& \text { - Pelleting of debris } \\
& (2500 \times \mathrm{xg}, 15 \mathrm{~min})
\end{aligned}
$$

$\sum \frac{2.5 \text { hours }}{30 \mathrm{~min}}$

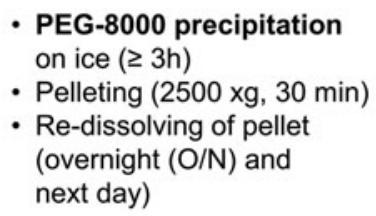

- PEG-8000 precipitation on ice $(\geq 3 \mathrm{~h})$

- Pelleting (2500 xg, $30 \mathrm{~min})$

- Re-dissolving of pellet (overnight $(\mathrm{O} / \mathrm{N})$ and next day)

\section{- Preparation of iodixanol gradient \\ - Ultracentrifugation \\ (1 h $15 \mathrm{~min}, 350,000 \mathrm{xg}$ )}

$\sum \frac{\mathrm{O} / \mathrm{N} \text { incubation }}{15 \mathrm{~min}}$

$14 \mathrm{~mL}$ AAV solution

$9 \mathrm{~mL} 15 \%$ iodixanol $6 \mathrm{~mL} 25 \%$ iodixanol $5 \mathrm{~mL} 40 \%$ iodixanol $5 \mathrm{~mL} 40 \%$ iodixanol
$5 \mathrm{~mL} 54 \%$ iodixanol

$\sum \frac{2 \text { hours }}{30 \mathrm{~min}}$

- Concentration/formulation (2 ultrafiltration cycles, $1000 \mathrm{xg}$ ) until desired final volume achieved

- Sterile-filtration

- Aliquoting, snap-freezing, storage
- Open $\mathrm{VH}$, collect following $3.5 \mathrm{~mL}$ (AAV; upper $54 \%$ and lower $40 \%$ phase)

- Puncture at top (ventilation hole, $\mathrm{VH}$ )

- Puncture at bottom (close $\mathrm{VH}$ with finger)

- Open VH, discard first $4 \mathrm{~mL}$ ( $54 \%$ phase)
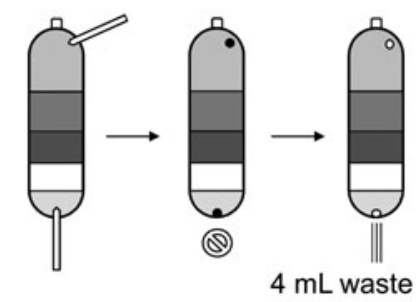

III

$3.5 \mathrm{~mL}$ AAV

$\sum 15 \mathrm{~min}$

$\sum \frac{30 \min }{30 \min }$

Figure 4. Process steps and time requirement for AAV "downstream" processing at $4,000 \mathrm{~cm}^{2}$ production scale. Shown are key process steps and the main time-determining centrifugation and incubation phases for the processes of cell harvest and lysis, pre-purification by PEG precipitation, main purification by an iodixanol step gradient and ultrafiltration, including information on process time requirement (white arrows), and actual hands-on time (black arrows). Together with Fig. 1b, this scheme illustrates the whole "one vial per CELLdisc" AAV large-scale production process. 
capsid variant in total. Finally, the pooled products of all 14 batches were also analyzed by SDS-PAGE, endotoxin measurements, cryo electron microscopy, and sequencing.

AAV2-BR1-CAG-eGPF average yield across 14 batches (two CELLdiscs each) was $8.41 \times 10^{13} \mathrm{VG}$, corresponding to $1.1 \times 10^{14} \mathrm{VG} / \mathrm{m}^{2}$ and approximately $5.7 \times 10^{4}$ VG per cell (based on an average of 728 million cells per CELLdisc at the time of transfection), with some operator dependent differences observed for batches \#7-12, however not exceeding a factor of two, which is within a usual range for AAV production (Fig. 5a). For AAV2-L1CMV-eGFP, the average yield was $2.5 \times 10^{13} \mathrm{VG}$, corresponding to $3.12 \times 10^{13} \mathrm{VG} / \mathrm{m}^{2}$ and $1.6 \times \mathrm{VG}$ per cell (Fig. 5b). In total, $6.1 \times 10^{14}$ and $1.2 \times 10^{15}$ purified vector genome-containing particles were produced for AAV2-L1 and -BR1, respectively. Notably, the observed productivity is well in line with reported wild-type AAV2 productivity using transfected, adherently grown HEK-293 cells, ${ }^{18,26}$ including recent reports using optimized plasmid designs for manufacturing in "cell factories" and the "iCELLis Nano" system $\left(2.2-4.7 \times 10^{14} \mathrm{VG} / \mathrm{m}^{2}\right) .{ }^{16}$
After pooling all runs, the final product was further analyzed by SDS-PAGE, confirming high purity and the expected 1:1:10 ratio of VP1:VP2:VP3 (Fig. 5c). Moreover, predominantly filled AAV vector particles were observed by cryo electron microscopy (Fig. 5d). Manual counting of full and emptyappearing particles revealed an empty particle ratio of $5.6 \%$ for AAV2-BR1 (based on a total of 550 counted particles) and $4.8 \%$ for AAV2-L1 (950 counted particles), which is a significant improvement over the previous observation of approximately 19\% empty particles using a more simplistic iodixanol protocol and, in particular, slightly less stringent gradient harvest criteria. ${ }^{4}$ Specifically, by now systematically discarding the first $4 \mathrm{~mL}$ outflow and harvesting the following $3.5 \mathrm{~mL} \mathrm{AAV} \mathrm{solution}$ (see Fig. 4) as opposed to harvesting based on changes in $340 \mathrm{~nm}$ absorbance measurements, as previously suggested, ${ }^{4,18}$ the amount of empty particles in iodixanol-purified preparations could be strongly decreased. The products were also confirmed to be free of endotoxins $(<5$ endotoxin units/mL). Finally, next-generation sequencing analysis (GeneWerk $\mathrm{GmbH}$ ) based on the approach
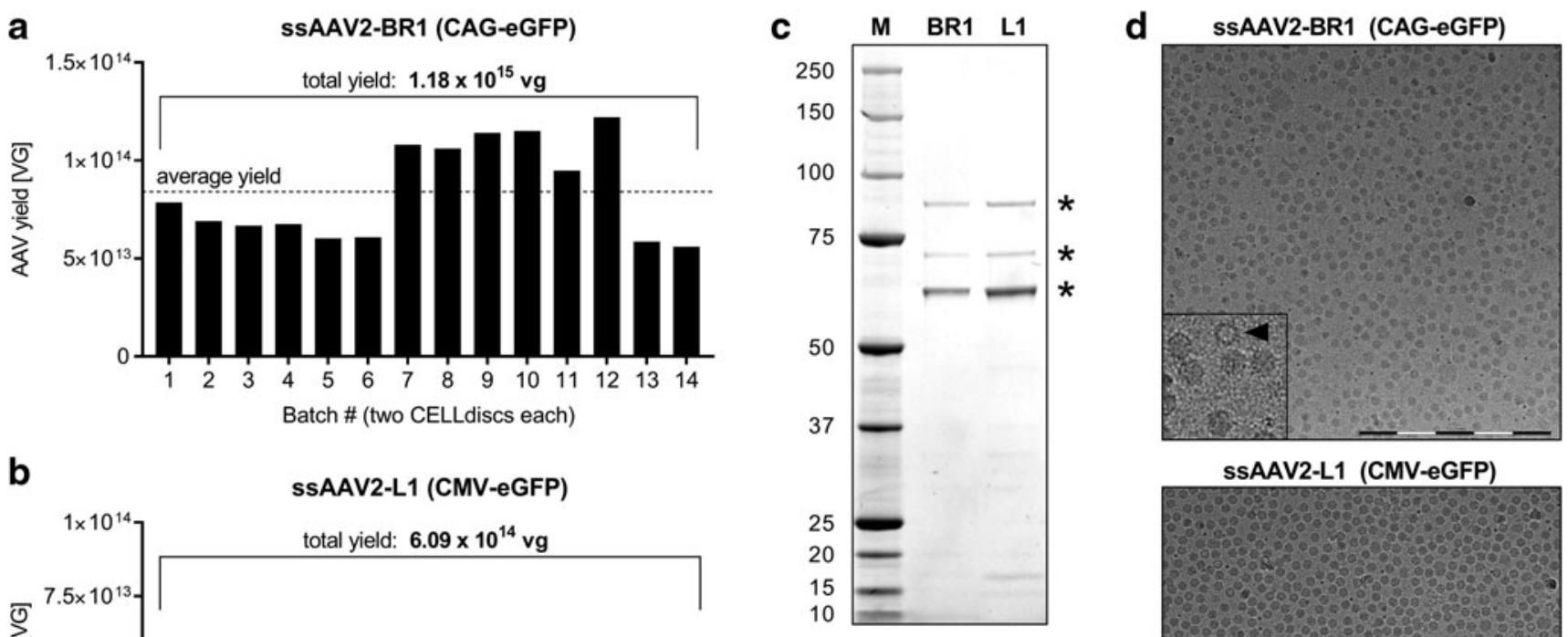

b

SSAAV2-L1 (CMV-eGFP)

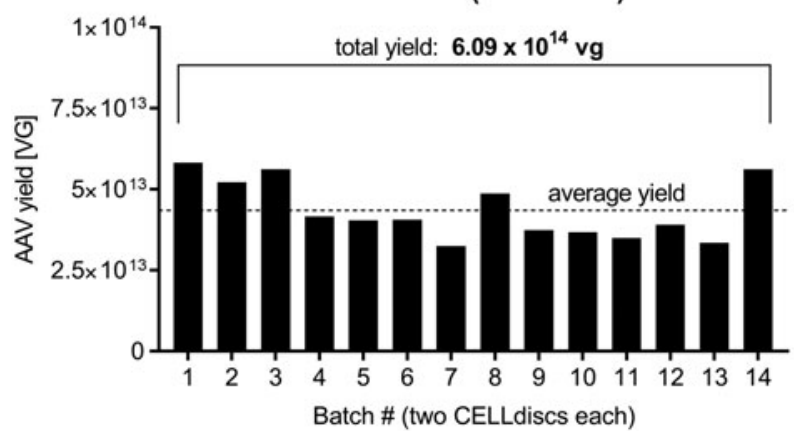

SSAAV2-L1 (CMV-eGFP)

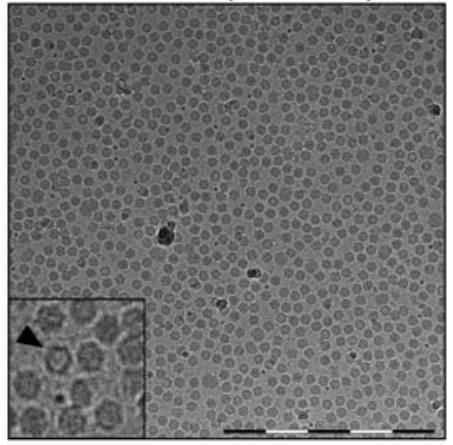

Figure 5. AAV2-BR1 and AAV2-L1 large-scale production (11.2 $\mathrm{m}^{2}$ each) using the one vial per CELLdisc strategy. For each of 14 batches per capsid variant, two CELLdiscs were seeded using frozen high-density $\left(60 \times 10^{6}\right)$ cell stocks. Following the purification protocol outlined in Fig. 4, (a) AAV2-BR1 and (b) AAV2-L1 vector batches were quantified by qPCR and AAV vector yields are shown in VG. The average yield across 14 batches is illustrated by the dashed line. (c) Vector purity was assessed by sodium dodecyl sulfate/SYPRO Ruby staining $\left(5 \times 10^{10} \mathrm{VG} / \mathrm{lane}\right)$. Asterisks: VP1 (87 kDa), VP2 (73 kDa), VP3 (62 kDa). (d) AAV particles were visualized by cryo electron microscopy to determine empty-to-full particle ratio. Insets exemplarily show threefold magnifications of empty (arrowhead) and full particles. Scale bar: $500 \mathrm{~nm}$. 
previously introduced by Lecomte et al. was performed. ${ }^{27}$ Besides revealing moderate nucleic acid contamination, originating from rep/cap plasmids (AAV2-BR1: 0.98\%; AAV2-L1: 0.28\%), helper plasmids $(0.96 \%, 0.37 \%)$, or the host cell genome $(<0.01 \%$ in both cases $)$, sequencing successfully confirmed the integrity of the packaged viral genome sequence.

Of note, whereas for the dish-based production of each vector at similar scale (i.e., $11.2 \mathrm{~m}^{2}$ ) seven hundred and thirty-six $15 \mathrm{~cm}$ dishes would have been necessary, in turn requiring preparatory culture expansion to a total of 90-140 T175 flasks, this process was realized using 28 vials of frozen cells together with 28 CELLdiscs. In summary, this streamlined process (Figs. $1 \mathrm{~b}$ and 4 ) allowed for the production of high-titer AAV vectors of high purity that qualify for subsequent use in large-animal biodistribution studies. Based on its successful performance, this process is now routinely used in the authors' lab for the production of AAV vectors. While at first sight the additional expenses for the custom production of high-density frozen instant cell stocks seem to increase overall manufacturing costs, the savings in cell culture material, media, and, importantly, hands-on time rapidly compensate for this presumed disadvantage. This production strategy will therefore be of interest for academic and industrial labs alike. Finally, in addition to CELLdiscs, this approach might also be used to optimize manufacturing processes based on different culture systems, including alternative, commonly used multilayered chambers, such as CellSTACKs and Cell Factories.

\section{CONCLUSIONS}

The present work investigated the idea of using frozen high-density HEK-293 cell stocks as a direct, ready-to-seed source of producer cells for recombinant AAV vector production. The study demonstrates that productivity in cells directly seeded from frozen stocks is equal to conventionally cultured and passaged cells, and that this production strategy can be combined with the multilayered CELLdisc system to scale up AAV production. Using this "one vial per CELLdisc" approach, it was possible to produce high-titer $\left(0.6-1.2 \times 10^{15}\right)$ AAV2-BR1 and -L1 batches for a large-animal study in as little as 4 weeks in a standard laboratory.

Besides a drastic reduction in cell culture work, this approach enables standardizing AAV manufacturing to low-passage, high-viability cells and provides the additional advantage of timely flexible initiation of production runs. This strategy will therefore be of high value for many labs performing AAV production.

\section{AUTHOR DISCLOSURE}

All authors are employees of Boehringer Ingelheim Pharma GmbH \& Co. KG.

\section{REFERENCES}

1. Hastie E, Samulski RJ. Adeno-associated virus at 50: a golden anniversary of discovery, research, and gene therapy success-a personal perspective. Hum Gene Ther 2015;26:257-265.

2. Strobel B., Duechs MJ., Schmid R. et al. Modeling pulmonary disease pathways using recombinant adeno-associated virus 6.2. Am J Respir Cell Mol Biol 2015;53:291-302

3. Aschauer DF, Kreuz S, Rumpel S. Analysis of transduction efficiency, tropism and axonal transport of AAV serotypes 1, 2, 5, 6, 8 and 9 in the mouse brain. PLoS One 2013;8:e76310.

4. Strobel B, Miller FD, Rist W, et al. Comparative analysis of cesium chloride- and iodixanol-based purification of recombinant adeno-associated virus (AAV) vectors for preclinical applications. Hum Gene Ther Methods 2015;26:147-157.

5. Zolotukhin S, Byrne BJ, Mason E, et al. Recombinant adeno-associated virus purification using novel methods improves infectious titer and yield. Gene Ther 1999;6:973-985.

6. Strobel B, Klauser B, Hartig JS, et al. Riboswitchmediated attenuation of transgene cytotoxicity increases adeno-associated virus vector yields in HEK-293 cells. Mol Ther 2015;23:1582-1591.

7. Maunder HE, Wright J, Kolli BR, et al. Enhancing titres of therapeutic viral vectors using the transgene repression in vector production (TRiP) system. Nat Commun 2017;8:14834.

8. Reid CA, Boye SL, Hauswirth WW, et al. miRNAmediated post-transcriptional silencing of transgenes leads to increased adeno-associated viral vector yield and targeting specificity. Gene Ther 2017:24:462-469.

9. Grimm D., Büning H. Small but increasingly mighty: latest advances in AAV vector research, design, and evolution. Hum Gene Ther 2017;28:10751086.

10. Robert M-A, Chahal PS, Audy A, et al. Manufacturing of recombinant adeno-associated viruses using mammalian expression platforms. Biotechnol J 2017;12.

11. Liu YL, Wagner $\mathrm{K}$, Robinson $\mathrm{N}$, et al. Optimized production of high-titer recombinant adenoassociated virus in roller bottles. BioTechniques 2003;34:184-189.
12. Ayuso E, Mingozzi F, Montane J, et al. High AAV vector purity results in serotype- and tissueindependent enhancement of transduction efficiency. Gene Ther 2010;17:503-510.

13. Zolotukhin S, Potter M, Zolotukhin I, et al. Production and purification of serotype 1, 2, and 5 recombinant adeno-associated viral vectors. Methods San Diego Calif 2002;28:158-167.

14. Potter M, Lins B, Mietzsch $M$, et al. A simplified purification protocol for recombinant adenoassociated virus vectors. Mol Ther Methods Clin Dev 2014;1:14034

15. Piras BA, Drury JE, Morton CL, et al. Distribution of AAV8 particles in cell lysates and culture media changes with time and is dependent on the recombinant vector. Mol Ther Methods Clin Dev 2016;3:16015.

16. Emmerling VV, Pegel A, Milian EG, et al. Rational plasmid design and bioprocess optimization to enhance recombinant adeno-associated virus (AAV) productivity in mammalian cells. Biotechnol J 2016;11:290-297.

17. Powers AD, Piras BA, Clark RK, et al. Development and optimization of AAV hFIX particles by transient 
transfection in an iCELLis $\left({ }^{\circledR}\right)$ fixed-bed bioreactor. Hum Gene Ther Methods 2016;27:112-121.

18. Lock M, Alvira M, Vandenberghe LH, et al. Rapid, simple, and versatile manufacturing of recombinant adeno-associated viral vectors at scale. Hum Gene Ther 2010;21:1259-1271.

19. Grieger JC, Soltys SM, Samulski RJ. Production of recombinant adeno-associated virus vectors using suspension HEK293 cells and continuous harvest of vector from the culture media for GMP FIX and FLT1 clinical vector. Mol Ther 2016;24:287-297.

20. Ai J, Ibraheim R, Tai PWL, et al. A scalable and accurate method for quantifying vector genomes of recombinant adeno-associated viruses in crude lysate. Hum Gene Ther Methods 2017;28:139-147.

21. Körbelin J, Dogbevia G, Michelfelder S, et al. A brain microvasculature endothelial cell-specific viral vector with the potential to treat neurovascular and neurological diseases. EMBO Mol Med 2016;8: 609-625.

22. Körbelin J, Sieber T, Michelfelder S, et al. Pulmonary targeting of adeno-associated viral vectors by next-generation sequencing-guided screening of random capsid displayed peptide libraries. Mol Ther J Am Soc Gene Ther 2016;24:1050-1061.

23. Vandenberghe $L H$, Xiao $R$, Lock $M$, et al. Efficient serotype-dependent release of functional vector into the culture medium during adeno-associated virus manufacturing. Hum Gene Ther 2010;21: 1251-1257.

24. Drouin LM, Lins B, Janssen M, et al. Cryo-electron microscopy reconstruction and stability studies of the wild type and the R432A variant of adenoassociated virus type 2 reveal that capsid structural stability is a major factor in genome packaging. J Virol 2016;90:8542-8551.
25. Crosson SM, Dib P, Smith JK, et al. Helper-free production of laboratory grade AAV and purification by iodixanol density gradient centrifugation. Mol Ther Methods Clin Dev 2018;10:1-7.

26. Aucoin MG, Perrier M, Kamen AA. Critical assessment of current adeno-associated viral vector production and quantification methods. Biotechnol Adv 2008;26:73-88.

27. Lecomte $E$, Tournaire $B$, Cogné $B$, et al. Advanced characterization of DNA molecules in rAAV vector preparations by single-stranded virus nextgeneration sequencing. Mol Ther Nucleic Acids 2015;4:e260

Received for publication November 28, 2018; accepted after revision January 26, 2019.

Published online: January 28, 2019. 